AperTO - Archivio Istituzionale Open Access dell'Università di Torino

\title{
Binding is responsible for exceptional hardness in polyethylene/silicalite nanocomposite materials
}

\section{This is the author's manuscript}

Original Citation:

Availability:

This version is available http://hdl.handle.net/2318/156707

since

Published version:

DOI:10.1039/C4CE01513D

Terms of use:

Open Access

Anyone can freely access the full text of works made available as "Open Access". Works made available under a Creative Commons license can be used according to the terms and conditions of said license. Use of all other works requires consent of the right holder (author or publisher) if not exempted from copyright protection by the applicable law. 


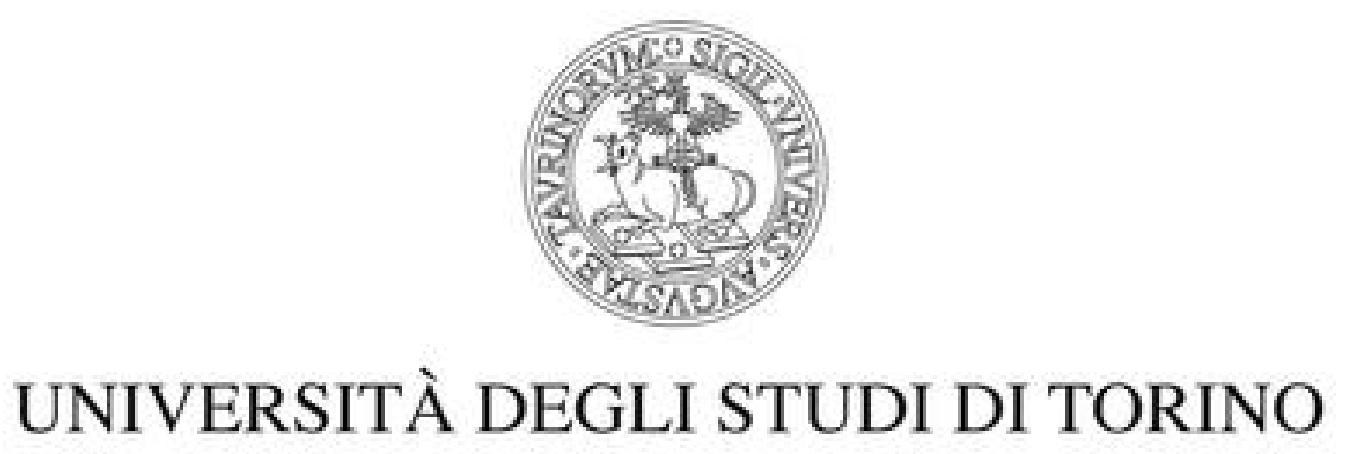

This is an author version of the contribution published on:

\section{Lorenzo Maschio}

Binding is responsible for exceptional hardness in polyethylene/silicalite nanocomposite materials

CRYSTENGCOMM (2014) 16

DOI: $10.1039 / \mathrm{C} 4 \mathrm{CE} 01513 \mathrm{D}$

The definitive version is available at:

http://xlink.rsc.org/?DOI=C4CE01513D 


\section{Binding is responsible for exceptional hardness in}

\section{Polyethylene/silicalite nanocomposite materials.}

Lorenzo Maschio

Dipartimento di Chimica and NIS (Nanostructured Interfaces and Surfaces) Centre - Università di Torino, Italy

Via P. Giuria 5, I-10125 Torino, Italy

e-mail: lorenzo.maschio@unito.it

\footnotetext{
ABSTRACT: Polyethylene/silicalite nanocomposites potentially represent a class of materials with extremely interesting mechanical properties. However, in order to outline a strategy for controlling their properties, a deep understanding of the physical phenomena taking place at an atomistic scale is required. Ab initio quantum mechanical simulations presented in this work show that by increasing or decreasing binding energy between the two moieties, the hardness of the material can be tuned at will. Moreover, residual internal stresses after synthesis significantly deteriorate mechanical properties, and a way to release them must be found.
} 
Nanocomposite materials with high crystallinity are rising as one of the most promising classes of new materials. ${ }^{1-3}$ Among them, PolyEthylene-SILicalite (PESIL) has particularly interesting features: ${ }^{3}$ it exhibits hardness comparable to that of quartz despite its considerably lower density, and shows negative thermal expansion - which makes it ideal for areospace applications. Recently synthesized and characterized by an Italian/French team, the composite can be obtained through polymerization of precursors inside the cavities of the $\mathrm{SiO}_{2}$ framework.

Such an interesting material calls for more attention. In particular, how can one tune and further improve its properties? A deep understanding of the underlying microscopic physico-chemical processes that trigger the macroscopic features of the material can be key in this direction.

The idea of polymer/silica nanocomposites is definitely not new. For a good review the reader can refer to Zou et al. ${ }^{4}$ Nevertheless, these materials generally are built from amorphous building blocks or at least have a low degree of crystallinity. Concerning the mechanical properties of Zeolite frameworks, some literature is relevant to the present study. A recent systematic computational study by Coudert ${ }^{5}$ has unveiled relationships between the zeolite density and sheer modulus/Young modulus but did not focus on silicalite. Older studies by Astala et al. ${ }^{6}$ reported computed elastic properties of several frameworks including silicalite, whose comparison with experimental data was however controversial. Li et al. ${ }^{7}$ have investigated experimentally zeolite structures with low dielectric constant and measured elastic constants as a function of porosity.

In this work show that the hardness of PESIL can be increased both by increasing the polyethylenesilicalite binding by chemical substitution or by rearrangement of the polymeric chains - that are subject to mechanical strain originated during in situ polymerization.

Simulations allowed to carry out experiments that are not possible in practice, such as to study the two moieties of the system separately and as a whole. Despite the wide range of applicability of modern $a b$ initio methods ${ }^{8,9}$ such theoretical studies are not yet common in the study of nanocomposite materials. 
Two main reasons for that are the unavailability, until recently, of nanocomposites with high crystallinity, and the relatively high computational costs.

The aim of this work is to answer fundamental questions about PESIL: which are the forces into play? How can the equilibrium be shifted in one direction or another? Is there a binding between the two moieties, or are they kept together solely by steric constraint? Determination of the coordinates of hydrogen atoms - which cannot be determined through XRD measurements - is an important goal too.

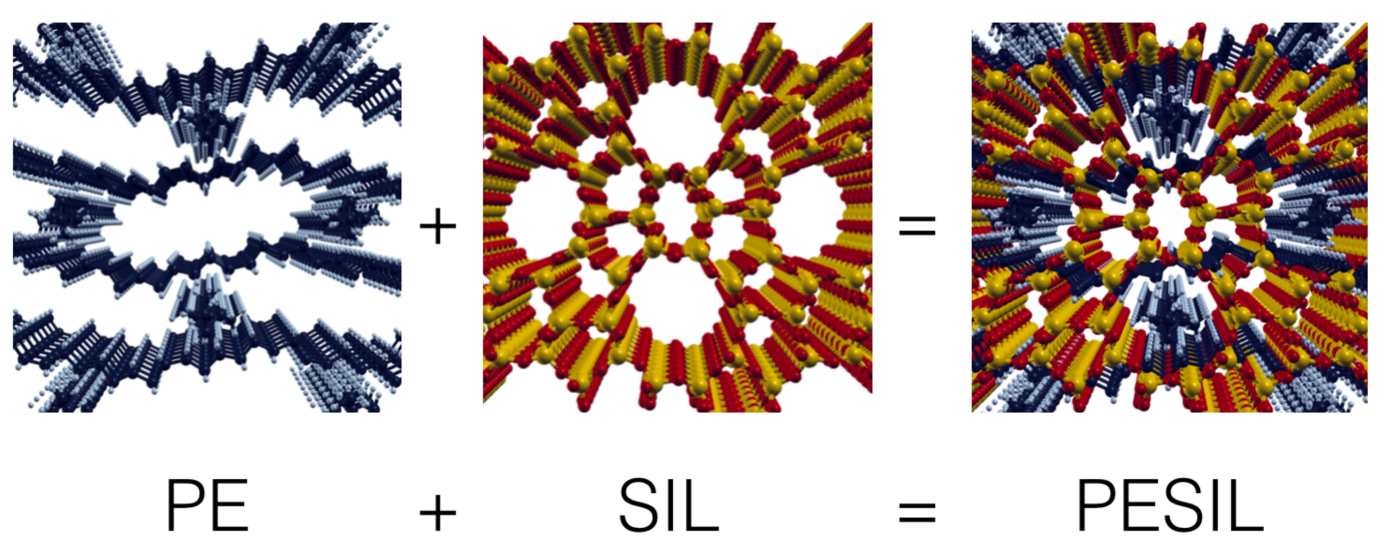

Figure 1 : The PESIL nanocomposite material is obtained from polymerization of polyethylene (PE) inside the cages of a silicalite (SIL) framework.

To answer these questions one can divide the two components of the material, while keeping the geometry fixed, and investigate each of them separately through calculations. In Figure 1 the two moieties of PESIL are depicted, together with the full structure. In the following these will be referred to as "PE", "SIL", and "PESIL".

As a first step, starting from the structure published in Ref. 3, I obtained the equilibrium position of hydrogen atoms. This was done inserting "by hand" hydrogen atoms in the PE (polymer alone) structure, and optimizing only the position of such atoms (that is, keeping frozen the position of carbons). Then I 
re-optimized the whole PESIL structure, obtaining a lattice parameter of $a=20.16, b=19.65, c=13.70$, with a cell volume of $5431 \AA^{3}$, extremely close to the experimental one of $5380 \AA^{3}$ (at $150{ }^{\circ} \mathrm{K}$ ). The full optimized geometry is reported in the supplementary material.

Next, I evaluated the Bulk modulus; to do that, one must compute the total unit cell energy of the structure relaxed at different volumes, ${ }^{10}$ and fit the curve by a given equation of state ( the Poirier-Tarantola logarithmic formula ${ }^{11}$ was here adopted). The corresponding curve is reported in panel a) of Figure 2. The computed bulk modulus is $27.9 \mathrm{GPa}$ - remarkably close to the experimental value of $26.7 \mathrm{GPa}-$ represents a further evidence that the structural model faithfully represents the real system.

The geometries used for computing the Bulk modulus are not only a by-product of the calculation, but can be useful in order to have a closer look at what is going on. The binding of PESIL, and the individual contributions to it, computed at each geometry are reported in Figure 2 panel a). Binding energy is evaluated as:

$\mathrm{E}^{\mathrm{BIND}}=\mathrm{E}^{\mathrm{PESIL}}-\left(\mathrm{E}^{\mathrm{PE}}+\mathrm{E}^{\mathrm{SIL}}\right)$ 
a)

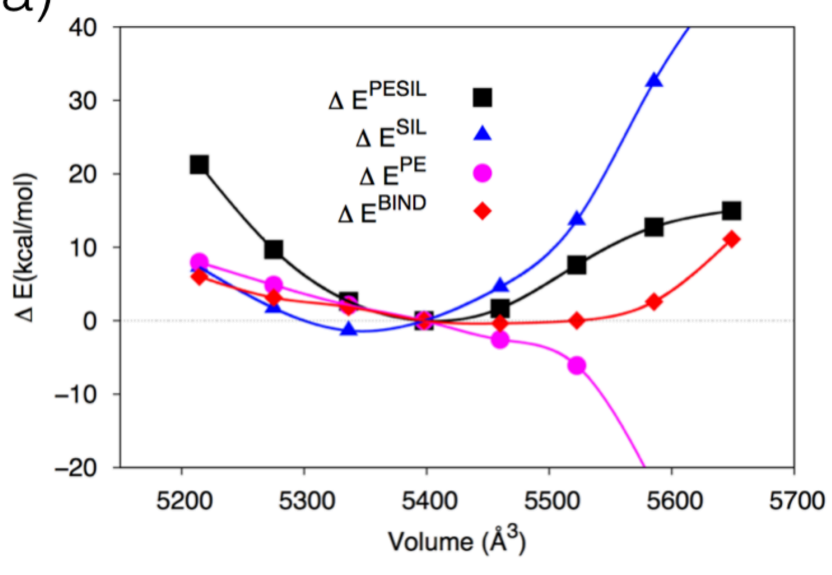

c)

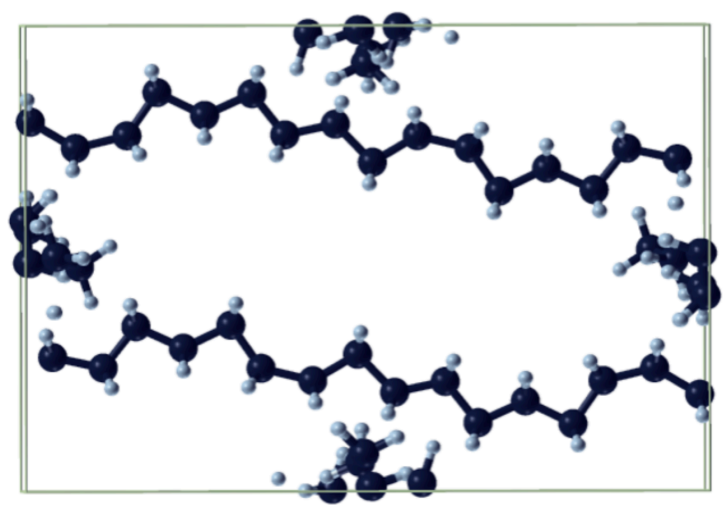

b)

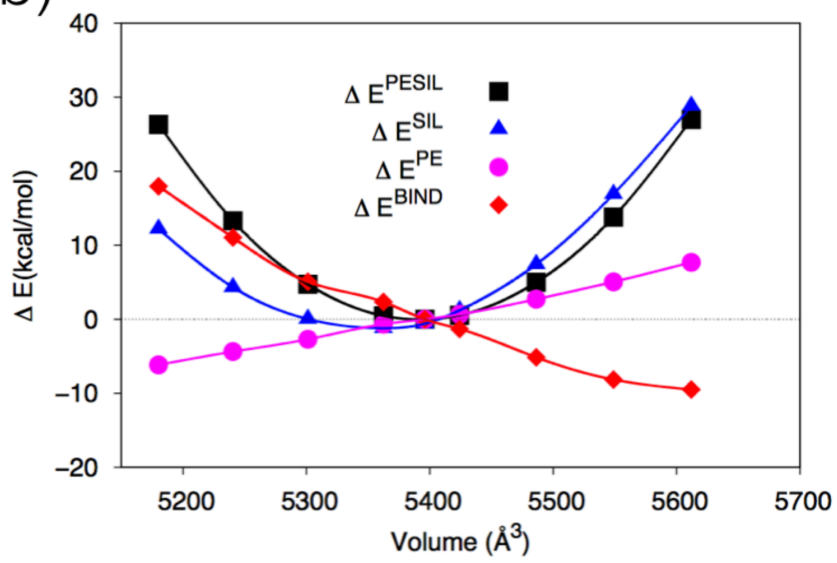

d)

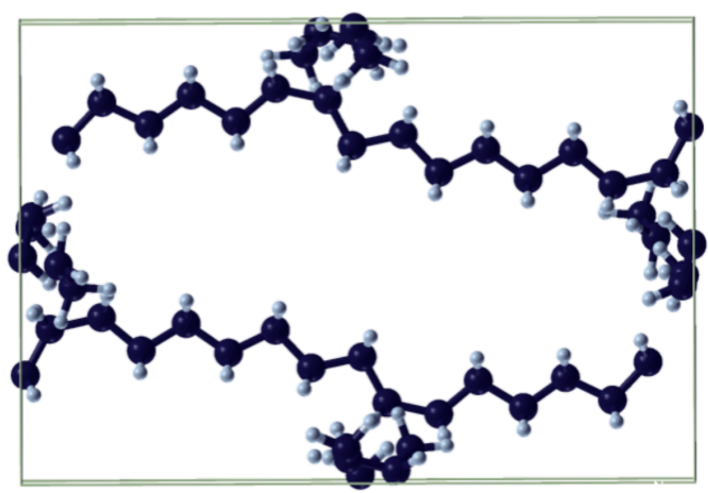

Figure 2 Energy decomposition of contributions to the total energy of PESIL before (panel a) and after (panel b) relaxation of strains in the polymeric (PE) moiety, reported as a function of the unit cell volume. In panels c) and d) changes in the conformation of the polymeric chain, before and after relaxation respectively, are clearly visible.

The value obtained for PESIL is $\mathrm{E}^{\mathrm{BIND}}=-169.3 \mathrm{kcal} / \mathrm{mol}$ per unit cell, at equilibrium geometry, which shows the interaction between PE and SIL is indeed attractive.

Surprisingly, the PE moiety seems to be longing for an expansion of the lattice, while the SIL moiety alone is essentially aiming to a slightly more contracted geometry ( the experimental volume of silicalite is $5325 \AA^{3}$ ). This finding is surprising since, as already observed, confined PE is highly strained and 
should pull towards more compact volumes. ${ }^{3}$ The binding energy also slightly drives towards more contracted geometry.

It is evident from inspection of Fig. 2a that, at large volumes, stabilization of the polymeric moiety with respect to the equilibrium geometry is particularly large. By closer inspection of the geometries, it can be seen that this extra stabilization is due to a rearrangement of the polymer inside the cages; by subsequent re-optimization of the whole structure starting from the most expanded geometry of the previous curve, a new, more stable equilibrium geometry is obtained: it has nearly the same lattice parameters as before $\left(a=20.00, b=19.74, c=13.66 \mathrm{Vol}=5396.09 \AA^{3}-\right.$ only slightly more contracted $)$ but relative stability about $75 \mathrm{kcal} / \mathrm{mol}$ (per unit cell) lower. Such structure - relative to the PE moiety — is depicted in panel d) of Fig. 2 (compared to the previous one, panel c) of the same figure), and its geometry is reported in supplementary material. The geometry of SIL framework is virtually unchanged.

In other words, once channels are large enough, the polymeric chains (that formed after polymerization of ethylene gas) have enough room to rearrange in a more comfortable configuration, releasing rotational and conformational strains, and this affects the macroscopic properties of the material (vide infra).

In panel b) of Fig. 2 the decomposition of the contributions to the binding for the new geometries is reported. Now both PE and SIL moieties drive towards smaller volumes, while interaction between the two is maximized at larger lattice parameters. Most importantly, the potential curve due to deformation is now steeper, leading to a computed bulk modulus of $43.0 \mathrm{GPa}$. As regards this curve, all points at small and large volumes have the same conformation, and no further movement of the polymeric chain is observed.

The new structure is, according to the calculations, harder than the experimentally studied one, while having the same density. The reason why this structure was not observed, probably lies in the not small activation barrier (estimated about $15-20 \mathrm{kcal} / \mathrm{mol}$ ). It is however reasonable to suppose that this barrier can be overcome through thermal treatment, of mechanical treatment, or a combination of both. 
Even more interestingly, in both structures the binding energy drives towards less dense and harder structures. This is an important indication in the search for new members of this class of nanomaterials with improved properties: by chemical substitution of some atoms, either in the silicalite framework or in the polymeric precursors, one might aim at a stronger binding between the two components, leading to a shift of the curves in Fig. 2b) towards the right hand side.

In summary, calculations indicate that a harder, more stable form of PESIL can be obtained. It can be concluded that, as far as polymer/zeolite composites are considered, the polymeric conformation obtained right after synthesis might not be the most stable one, but this can be optimized if the polymeric moiety is given some freedom to rearrange.

The idea to increase the dispersive interaction between the two moieties, for instance through substitutions of ions in the silicalite structure, to obtain a harder material constitutes a stimulating hypothesis to be verified in future studies. Possibilities of different framework structures or different polymeric chains are also worth to be investigated in a systematic way.

\section{COMPUTATIONAL METHODS}

Calculations at the DFT (Density Functional Theory) level have been performed using the CRYSTAL14 code. $^{12,13}$ A hybrid B3LYP functional corrected for dispersion effects ${ }^{14}$ was adopted, and a Gaussian basis set (reported in detail in Supplementary material) was used. Binding energies are corrected for basis set superposition error by standard counterpoise method. Images have been obtained using the XCrySDen software. $^{15}$

\section{SUPPORTING INFORMATION}

Supporting information is available, containing the full information about equilibrium structures, basis set adopted, and additional pictures of optimized geometries.

\section{AUTHOR INFORMATION}




\section{Corresponding Author}

*lorenzo.maschio@unito.it

\section{REFERENCES}

1. Laird E. D. \& Li C. Y. Structure and Morphology Control in Crystalline Polymer-Carbon Nanotube Nanocomposites Macromolecules 46, 2877-2891 (2013).

2. Liao Y. et al. Facile Synthesis of High-Crystallinity Graphitic Carbon/Fe3C Nanocomposites As Counter Electrodes for High-Efficiency Dye-Sensitized Solar Cells ACS Appl. Mater. Interfaces 5 (9), 3663-3670 (2013).

3. Santoro, M., Gorelli, F. A., Bini, R., Haines, J. \& van der Lee A. High-pressure synthesis of a polyethylene/zeolite nano-composite material Nature Communications 4:1557 (2013)

4. H. Zou, S. Wu, J. Shen, Polymer/Silica Nanocomposites: Preparation, Characterization, Properties, and Applications, Chem Rev. 108, 3893-3957 (2008)

5. Coudert F.-X. Systematic investigation of the mechanical properties of pure silica zeolites: stiffness, anisotropy, and negative linear compressibility. Phys. Chem. Chem. Phys. 15, 16012 (2013).

6. R. Astala, S. M. Auerbach, P. A. Monson, Density Functional Theory Study of Silica Zeolite Structures: Stabilities and Mechanical Properties of SOD, LTA, CHA, MOR, and MFI, J. of Phys. Chem. B 108 (26), 9208-9215 (2004)

7. Z. Li, M. C. Johnson, M. Sun, E. T. Ryan, D. J. Earl, W. Maichen, J. I. Martin, S. Li, C. M. Lew, J. Wang, M. W. Deem, M. E. Davis, Y. Yan, Mechanical and Dielectric properties of Pure-Silica-Zeolite Low- $k$ Materials, Angew. Chem. Int. Ed. 45, 6329-6332 (2006) 
8. Tan, J., Civalleri, B., Lin, C., Valenzano, L., Galvelis, R. Chen, P., Bennett, T. D., MellotDraznieks, C., Zicovich-Wilson, C. \& Cheetham , A. K. Exceptionally Low Shear Modulus in a Prototypical Imidazole-Based Metal-Organic Framework Phys. Rev. Lett. 108, 095502 (2012).

9. Rimola, A., Aschi, M., Orlando R. \& Ugliengo P. Does Adsorption at Hydroxyapatite Surfaces Induce Peptide Folding? Insights from Large-Scale B3LYP Calculations. Journal of the American Chemical Society 134 (26), 10899-10910 (2012).

10. Perger, W. F., Criswell, J., Civalleri, B. \& Dovesi, R. Ab-initio calculation of elastic constants of crystalline systems with the CRYSTAL code. Comp. Phys. Commun. 180(10), 1753-1759 (2009).

11. Poirier, J. P. \& Tarantola, A. A logarithmic equation of state. Phys. Earth Planet Inter. 109, 1 (1998)

12. Dovesi, R. et al. "CRYSTAL14: A program for the ab initio investigation of crystalline solids" Int. J. Quantum Chemistry, 114(19), 1287-1317 (2014) , DOI: 10.1002/qua.24658

13. Orlando R., Delle Piane M., Bush I.J., Ugliengo P., Ferrabone M. \& Dovesi R. A new massively parallel version of CRYSTAL for large systems on high performance computing architectures. J Comput Chem. 33(28), 2276-84 (2012).

14. Grimme S., Semiempirical gga-type density functional constructed with a long-range dispersion correction. J. Comp. Chem. 27, 1787 (2006).

15. Kokalj, A. XCrySDen--a new program for displaying crystalline structures and electron densities, J. Mol. Graphics Modelling, 17, 176-179 (1999). 